\title{
Modelling of Methane Hydrate Formation and Dissociation using Residual Thermodynamics
}

\author{
Solomon Aforkoghene Aromada ${ }^{1}$, Bjørn Kvamme ${ }^{2}$ \\ 1Department of Physics and Technology, University of Bergen, Allegaten 55, 5007 Bergen, Norway; \\ *Solomon.Aromada@student.uib.no, saromada@gmail.com \\ 2Strategic Carbon LLC, Vestre Holbergsallmenningen 17, 5011 Bergen, Norway; kvamme@strategic-carbonllc.com
}

SNE 31(3), 2021, 143-150, DOI: 10.11128/sne.31.tn.10575 Received: March 10, 2021 (Selected EUROSIM 2019 Postconf. Publ.); Revised: August 30, 2021; Accepted: September 2, 2021 SNE - Simulation Notes Europe, ARGESIM Publisher Vienna ISSN Print 2305-9974, Online 2306-0271, www.sne-journal.org

Abstract. The available experimental data in literature for enthalpies of hydrate formation and dissociation are limited and often lacks relevant information required for interpretation. Commonly missing information include hydrate composition, hydration number, temperature and/or pressure data, and degree of super heating during dissociation of hydrate.

Clausius-Clapeyron equations used with measured or calculated hydrate formation pressure-temperature equilibrium data is the simplest indirect methods used for evaluating enthalpy change involved in phase transition during hydrate formation or dissociation. This approach involves over-simplifications. These oversimplifications make all the data based on Clausius-Clapeyron to be unreliable. And old data using Clapeyron do not have appropriate volume corrections. We therefore propose a thermodynamic scheme (residual thermodynamics approach) which does not have these limitations. This method is based on residual thermodynamics for all properties like equilibrium (pressure-temperature) curves, free energy change as thermodynamic driving force in kinetic theories and enthalpies of hydrate formation and dissociation. The pressure-temperature equilibrium curve obtained in this work agrees well with literature.

\section{Introduction}

Natural gas hydrates are non-stoichiometric crystalline inclusion compounds (ice-like substances) formed when hydrogen-bonded water molecules form three-dimensional solid cage-like structures with cavities which entrap suitably small sized molecules of certain gases and volatile liquids known as guest molecules, under the condition of high pressure and low temperature. Unlike ice, they exist above $273.15 \mathrm{~K}\left(0{ }^{\circ} \mathrm{C}\right)$. Lighter hydrocarbon components [1,2] and some inorganic gases $[3,4]$ are guest molecules that can form hydrate in their pure form.

A vast amount of natural occurring methane hydrates is distributed all over the world in the permafrost and in the oceans [5]. This huge amount of methane gas trapped in the naturally existing hydrate could be a potential source of unconventional energy. In a time when decarbonazation and the use of low-carbon energy resources have become exigent, successfully exploiting this huge amount of natural gas stored in form of hydrate will be important.

To produce this methane, any method that could be used will require supply of heat [6] to dissociate the methane hydrate. Therefore, a study of the heat of dissociation and formation of methane hydrate is important. Thus, it is essential to examine how best these heat (exothermic for formation and endothermic for dissociation) can be better evaluated. The amount of heat required for dissociation of the hydrate is the same amount that is released when the hydrate is form. The difference in representation is in the sign, negative for formation and positive for dissociation.

Hydrate formation is a complex exothermic process that involves competing phase transition mechanisms and routes where kinetics and thermodynamics play important role. The exothermic heat released (enthalpy of formation) during the phase transition is one of the most significant thermodynamic properties that we need for proper understanding of the phase transition process. This heat is either measured directly [7] by experiment or indirectly using Clausius-Clapeyron [8] or Clapeyron [9] modelling approaches. These approaches have some limitations and the results obtainable in literature often lack important information. 
For example, the available experimental data in literature for enthalpies of hydrate phase transition are limited and often lacks relevant information required for interpretation. Frequently missing information are composition of the hydrates, hydration number, temperature and/or pressure data, and degree of super heating required during dissociation of hydrate. ClausiusClapeyron equations used with measured or calculated hydrate formation pressure-temperature equilibrium data is the simplest indirect methods used for evaluating enthalpy change during hydrate phase transition. This approach involves over-simplifications. These oversimplifications make all the data based on Clausius-Clapeyron to be unreliable. And old data from Clapeyron do not have appropriate volume corrections.

Therefore, a consistent thermodynamic scheme, residual thermodynamics approach is proposed in this work and implemented based on the the trivial thermodynamic relationship between enthalpy change and free energy change. This method is based on using residual thermodynamics for all properties like equilibrium (pressuretemperature) curves, free energy change as thermodynamic driving force in kinetic theories and enthalpies of hydrate formation and dissociation. The approach eliminates the limitations.

The relevant modelling equations are presented, and simulations were performed. The results are discussed are compared with literature.

\section{Modelling of Hydrate Dissociation with Residual Thermodynamics}

Our modelling approach is summarized in this section. The free energy change for a specific hydrate phase transition can expressed as:

$$
\begin{aligned}
& \Delta G^{\left(H_{1}\right)}=x_{H_{2} O}^{H}\left(\mu_{H_{2} O}^{H}\left(T, P, \overrightarrow{x^{H}}\right)-\mu_{H_{2} O}^{\text {water }}(T, P, \vec{x})\right)+ \\
& \sum_{j} x_{j}^{H}\left(\mu_{j}^{H}\left(T, P, \overrightarrow{x^{H}}\right)-\mu_{j}^{g a s}\left(T, P, \overrightarrow{y^{g a s}}\right)\right)
\end{aligned}
$$

The superscript $H_{l}$ distinguishes the specific heterogeneous phase transition from other hydrate formation phase transitions. $T$ is temperature, $P$ is pressure. $x$ is mole-fraction in either liquid or hydrate (denoted with a subscript $H$ ) while $y$ is mole-fraction in gas (or liquid) hydrate former phase. $j$ is an index for hydrate formers.
Superscript water denotes water phase that is converted into hydrate. Generally, this is ice or liquid but, in this work, we only consider liquid water. $\mu$ is chemical potential. These chemical potentials are convenient in discussing other routes to hydrate formation and associated hydrate former chemical potentials since any variation in chemical potential of hydrate formers will lead to changes in hydrate compositions and hydrate free energies. This is fundamentally important since any assembly of molecules with unique density and composition is a unique phase. Liquid water chemical potential is calculated from the symmetric excess conventions as:

$$
\begin{gathered}
\mu_{\mathrm{H}_{2} \mathrm{O}}(T, P, \vec{x})=\mu_{\mathrm{H}_{2} \mathrm{O}}^{\text {pure } \mathrm{H}_{2} \mathrm{O}}(T, P)+ \\
R \cdot \operatorname{Tln}\left(x_{\mathrm{H}_{2} \mathrm{O}} \cdot \gamma_{\mathrm{H}_{2} \mathrm{O}}(T, P, \vec{x})\right) \approx \mu_{\mathrm{H}_{2} \mathrm{O}}^{\text {pure } \mathrm{H}_{2} \mathrm{O}}(T, P)+ \\
R . T \ln \left(x_{\mathrm{H}_{2} \mathrm{O}}\right)
\end{gathered}
$$

$$
\lim \left(\gamma_{\mathrm{H}_{2} \mathrm{O}}\right)=1.0 \text { when } x_{\mathrm{H}_{2} \mathrm{O}} \text { tends to unity }
$$

The focus here is to illustrate the complexity of multiple hydrate formation in systems of water and $\mathrm{CH}_{4}$. We used a simpler kinetic model which is more visible in terms of the various contributions to the hydrate phase transition dynamics. As such the approximation on the right-hand side of equation (2) is accurate enough for the purpose. The solubility of $\mathrm{CH}_{4}$ in water is small and the right-hand side will be close to pure water chemical potential. Chemical potential for water in the hydrate structure is given by [10]:

$$
\mu_{H_{2} O}^{H}=\mu_{H_{2} O}^{O, H}-\sum_{k=1,2} R T v_{k} \ln \left(1+\sum_{i} h_{i j}\right)
$$

in which $H$ denote hydrate and $O$ in the superscript on first term on right hand side means empty clathrate. These chemical potentials are readily available from model water (TIP4P) simulations [11]. The number of cavities per water, $v_{k}$ is $1 / 23$ for small cavities of structure I and 3/23 for large cavities. With $\mathrm{CH}_{4}$ as only the guest, $i$ is 1 in the sum over the canonical partition functions for small and large cavities.

$$
h_{k i}=e^{\beta\left[\mu_{k i}-\Delta g_{k i}\right]}
$$

The enthalpy change is trivially related to the corresponding free energy change by the thermodynamic relationship: 


$$
\frac{\partial\left[\frac{\Delta G^{\text {Total }}}{R T}\right]_{P, \vec{N}}}{\partial T}=-\left[\frac{\Delta H^{\text {Total }}}{R T^{2}}\right]
$$

The superscript total is introduced to also include the penalty of pushing aside the old phases.

Practically the total free energy change will be equation (2) plus the interface free energy times contact area between water and hydrate forming phase during the nucleation stage divided by number of molecules in the specific core size. Since critical nuclei sizes are small the whole particle can be considered as covered with water due to capillary forces. Above critical core size the penalty diminishes rapidly relative to the free energy benefits from (2).

$\frac{\partial\left[\frac{\mu_{H_{2} O}^{H}}{R T}\right]_{P, \widehat{N}}}{\partial T}=\frac{\partial\left[\frac{\mu_{H_{2} O}^{0, H}}{R T}\right]_{P, \widehat{N}}}{\partial T}-\left[\frac{\partial}{\partial T}\right]_{P, \vec{N}}\left[\sum_{k=1,2} v_{k} \ln \left(1+\sum_{i} h_{k i}\right)\right]$

For the liquid water phase in (2), as wcell as for the empty hydrate chemical potential on right hand side of equation (6) results are trivially obtained from [12] while the second term on right hand side is reorganized as:

$$
\left[\frac{\partial}{\partial T}\right]_{P, \bar{N}}\left[\sum_{k=1,2} v_{k} \ln \left(1+\sum_{i} h_{k i}\right)\right]=\left[\sum_{k=1,2} v_{k} \frac{\sum_{i}\left[\frac{\partial h_{k i}}{\partial T}\right]_{P \vec{N}}}{\left(1+\sum_{i} h_{k i}\right)}\right]
$$

And the derivatives of the cavity partition functions can be written as:

$$
\left[\frac{\partial h_{k i}}{\partial T}\right]_{P \bar{N}}=h_{k i}\left[-\frac{1}{R T^{2}}\left(\mu_{k i}-\Delta g_{k i}\right)+\frac{1}{R T}\left(\frac{\partial \mu_{k i}}{\partial T}-\frac{\partial \Delta g_{k i}}{\partial T}\right)\right]
$$

The partial derivatives in the last term on right hand side is numerically differentiated from the polynomial fits of [11].

$$
\begin{aligned}
& \frac{\partial\left[\frac{\mu_{H_{2} O}^{H}}{R T}\right]_{P \vec{N}}}{\partial T}=\frac{\partial\left[\frac{\mu_{H_{2} O}^{0, H}}{R T}\right]_{P \vec{N}}}{\partial T}+ \\
& {\left[\sum_{k=1,2} v_{k} \frac{\sum_{i} h_{k i}\left[\frac{1}{R T^{2}}\left(\mu_{k i}-\Delta g_{k i}\right)-\frac{1}{R T}\left(\frac{\partial \mu_{k i}}{\partial T}-\frac{\partial \Delta g_{k i}}{\partial T}\right)\right]}{\left[1+\sum_{i} h_{k i}\right]}\right]}
\end{aligned}
$$

$$
\begin{gathered}
H_{H_{2} O}^{0, H}=-R T^{2} \frac{\partial\left[\frac{\mu_{H_{2} O}^{H, 0}}{R T}\right]_{P \vec{N}}}{\partial T}+ \\
{\left[\sum_{k=1,2} v_{k} \frac{\sum_{i} h_{k i}\left[\frac{1}{R T^{2}}\left(\mu_{k i}-\Delta g_{k i}\right)-\frac{1}{R T}\left(\frac{\partial \mu_{k i}}{\partial T}-\frac{\partial \Delta g_{k i}}{\partial T}\right)\right]}{\left[1+\sum_{i} h_{k i}\right]}\right]}
\end{gathered}
$$

For liquid water, the enthalpy is even more trivially obtained by numerical differentiation of the polynomial fit of chemical potential as function of T given by [10].

In an equilibrium situation, chemical potential of the same guest in the two cavity types must be the same and these have to be equal to the chemical potential of the same molecule in the phase that it came from. For the heterogeneous case this means chemical potential of the molecule in gas (or liquid) hydrate former phase. But outside of equilibrium the gradients in chemical potentials as function of $T, P$ and mole-fractions have to reflect how the molecule behaves in the cavity.

Enthalpies for various guest molecules in the two types of cavities can be evaluated by Monte Carlo simulations along the lines described by [10-12] by sampling guest water interaction energies and efficient volumes from the movements of the guest molecules. That is:

$$
H_{k i}^{R}=U_{k i}^{R}+\left(z_{k i}-1\right) R T
$$

where $U$ is energy and superscript $R$ denote residual (interaction) contribution. $z_{k i}$ is compressibility factor for the guest molecule $i$ in cavity $k$. Consistent ideal gas values for the same interaction models that were applied in calculation of the residual values is trivial.

$$
z_{k i}=\frac{P V_{k i}}{k_{B} T}
$$


In which $k_{B}$ is Boltzmann's constant and is the excluded volume of a molecule of type $i$ in cavity of type $k$. This latter volume is calculated from the sampled volume of centre of mass movements plus the excluded volume due to water/guest occupation. Slightly more complex sampling and calculation for molecules which are not monoatomic (or approximated as monoatomic as methane) but still fairly standard $(6,7)$ and explicit discussion on this is not needed here. The derivative of the chemical potential of a guest molecule $i$ in cavity type $k$ with respect to temperature as needed in equation (9) is the negative of partial molar entropy for the same guest molecule and can be calculated according to:

$$
\left[\frac{\partial \mu_{k i}}{\partial T}\right]_{P \vec{N}}=\frac{\mu_{k i}-H_{k i}}{T}
$$

Equation (10) can then be rearranged into:

$$
\begin{gathered}
H_{H_{2} O}^{0, H}=-R T^{2} \frac{\partial\left[\frac{\mu_{H_{2} O}^{H, O}}{R T}\right]_{P \vec{N}}}{\partial T}+ \\
{\left[\sum_{k=1,2} v_{k} \frac{\sum_{i} h_{k i}\left[H_{k i}-\Delta g_{k i}+T \frac{\partial \Delta g_{k i}}{\partial T}\right]}{\left[1+\sum_{i} h_{k i}\right]}\right]}
\end{gathered}
$$

Residual enthalpies for hydrate former in a separate hydrate former phase are trivially given by:

$$
H_{k i}^{R}=-R T^{2} \sum_{i} y_{i}\left[\frac{\partial \ln \phi_{i}^{g a s}}{\partial T}\right]_{P, y_{j \neq i}}
$$

In which the same equation of state (SRK) is utilized as the one used for calculating fugacity coefficients for the chemical potentials.

\section{Methane Hydrate Equilibrium Pressure - Temperature}

Hydrate equilibrium pressures for methane hydrate formation were estimated for a temperature range of $273 \mathrm{~K}$ to $290 \mathrm{~K}$ as can be seen in Fig. 1. The estimates are compared with literature [9, 13] and there is a very good agreement even though we did not fit interaction parameters, which is not the priority here.

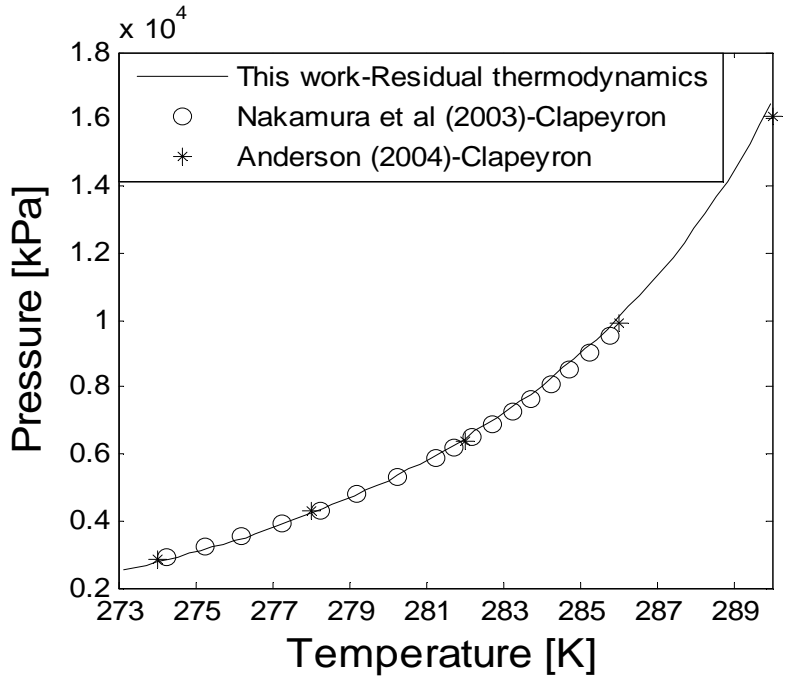

Figure 1: Estimated methane hydrate equilibrium pressures using residual thermodynamics (this work) compared with literature $[9,15]$.

The priority is to keep the statistical mechanical model free of adjustable parameters in all terms, together also with empty hydrate chemical potentials and chemical potentials for ice and liquid water.

\section{Enthalpies of Methane Hydrate Formation along Equilibrium Curve}

The experimental data available in literature for enthalpies of hydrate formation and dissociation are limited and often lacks significant information required for interpretation. Commonly missing information include hydrate composition, hydration number, temperature and/or pressure data, and degree of super heating in the course of dissociation of hydrate. Hydrate dissociation enthalpy are measured directly or evaluated indirectly. Calorimetry, NMR, Raman, pressure drop X-ray diffraction are some of the methods used for direct measurement. And for the indirect method,

Clapeyron and Clausius-Clapeyron equations are the approaches that are usually employed. The simplest indirect method is the Clausius-Clapeyron equation [8] and it is used with measured or calculated hydrate formation pressure-temperature equilibrium data. The simplifications in this approach limit the accuracy of results for higher pressures. Therefore, more recent studies use the original Clapeyron equation with various models for the volume changes associated with the phase transitions [9]. 
These oversimplifications make data based on Claussius-Clapeyron to be unreliable. In addition, the old data using Clapeyron do not have appropriate volume corrections. The data from Anderson involves very high filling fractions of the hydrate. Some of the calculated filling fractions reported by Anderson [9] seem very high, even up to $282 \mathrm{~K}$. And most calorimetry data do not have any measured filling fraction and often use a con

Therefore, there is a need for consistent and reliable enthalpies of hydrate formation or dissociation data, and that is why we propose the use of residual thermodynamics. This method is based on residual thermodynamics for all properties like equilibrium (pressure-temperature) curves, free energy change as thermodynamic driving force in kinetic theories, and enthalpies of hydrate formation and dissociation. This scheme is also not limited to heterogeneous hydrate formation from water, and a separate hydrate former phase. It can be used to evaluate associated enthalpy change in homogeneous hydrate formation from dissolved hydrate forming guest molecules in water. Even though we have applied the theory to one component (methane) because of the acceptable limit of work to be presented, there is no limitation in its application to other guest molecules and mixtures of hydrate formers (as we shall demonstrate in subsequent work), the formalism is written for mixtures. Another important advantage of this approach, unlike the Clapeyron method is that it can easily be extended to conditions outside of equilibrium as well as to other hydrate phase transitions. Applicable examples are enthalpy changes associated with hydrate forming from dissolved hydrate guest molecules in water, and the reverse process of hydrate dissociation to water under-saturated with guest molecules. Additional applicable hydrate phase transitions are nucleation of hydrate towards mineral surfaces. Our filling fractions seem realistic and reproduce equilibrium pressures as shown in Figure 1. Anderson [9] used a specific code. This code is based on fitting of also the difference between chemical potential of empty hydrate and water as well as associated fitting of several related differences needed to calculate chemical potential differences up to actual temperatures and pressures. Fitting fundamental properties like chemical potentials is by itself questionable.

Our estimates of enthalpy change for methane hydrate formation from pure methane and liquid water along the hydrate equilibrium $(\mathrm{P}, \mathrm{T})$ curve, that is three-phase coexistence conditions (liquid water, hydrate and gas simply represented as L-H-V) are presented in Figure 2 and have been calculated using residual thermodynamics.
In this figure, our intention is not to validate our scheme using these literature values. Based on all the limitations we have pointed out above, we do not expect our result to agree perfectly with literature. However, Nakamura et al. [12] results are closer to the results of our work compared to the other literature. The results estimated from Clapeyron approaches by Nakamura et al. [13] and Anderson [9] disagree significantly both in values and trend. There is a very wide difference or deviation in their results. The work of Nakamura et al. [13] even though it shows a very weak dependence on temperature till around $280 \mathrm{~K}$, follows similar trend with our work, therefore, we have Table 1 for easy comparison. Table 2 gives the results from using our scheme and some literature $[9,14,15]$. Kang et al. [15] estimated enthalpy change of phase transition at $274.15 \mathrm{~K}$ from isothermal micro-calorimeter experiment is only also close to result of this scheme at $274.10 \mathrm{~K}$. it is only $0.4 \%$ higher than the estimated values from the residual thermodynamic approach. The pressure at this temperature is missing in the paper of Kang et al. [15]. It is also important to keep in mind that also experiments have various limitations. Some of these are discussed by Kvamme et.al. [16]. Anderson's [9] results are the lowest and the trend is opposite to those of Nakamura et al. [13] and this work. Application of this scheme for $\mathrm{CO}_{2}$ guest molecule and the implication of enthalpy changes of hydrate phase transitions for simultaneous methane production from in-situ methane hydrates and storage of $\mathrm{CO}_{2}$ (zero-emission concept) as $\mathrm{CO}_{2}$ hydrate can be found in $[17,18]$.

Claussius approach, or simplified Claussius-Clapeyron approach, is generally not applicable for mixtures. At least not in the simple form used by Anderson [9] and others. For a pure component there is no composition dependency on either side of the co-existence curve. This changes the formal derivation of Claussius when changing to mixtures and might make it difficult to use. Hydrate is a mixed component and even the use of Clapeyron for hydrate is not straightforward. There are publications that formaluate a fugacity for hydrate, which at best is empirical. Fugacity is uniquely related to chemical potential on individual component basis.

To the best of our knowledge, there are no other models for enthalpy that can be utilized for gas mixtures [19, 20], and for different hydrate phase transitions like for instance hydrate formation from dissolved hydrate formers in water [21]. In principle there is no limit in the various hydrate formation possibilities. 
As long as chemical potential for water and hydrate formers can be calculated then the model cam be utilized. See for instance Kvamme et. al [22] for example of work in progress on hydrate formation from adsorbed methane in structured water towards mineral surfaces. And since the model is derived from Gibbs free energy model it is also consistent [19] and incorporates impact of component in water that affects chemical potentials for water, also on enthalpies.

In this work, hydration number was also estimated as given in Table 2 where the results from this work are compared with literature. The enthalpies are negative because hydrate formation is exothermic. The hydrate formation enthalpy is the heat of hydrate crystallization that must be transported out of the system, the system must lose this heat if the hydrate must form when every other condition favourable for hydrate to form is met. The heat transport is about 2-3 times [23] the magnitude of mass transport, that is more rapid. Heat transport limitation could lead to hydrate dissociation. These enthalpy values are the same for methane hydrate dissociation. But for hydrate dissociation, the values will be positive since heat is added to the system, or heat is required by the system for hydrate dissociation to proceed.

\section{Conclusion}

We used a consistent thermodynamic approach to evaluate the enthalpies of hydrate formation and dissociation and hydration number of methane hydrate. The methane hydrate equilibrium pressure-temperature curve estimated with this scheme agrees well with literature.
The results estimated from Clapeyron approaches by Nakamura et al. [12] and Anderson [8] disagree significantly both in values and trend. Nakamura et al. [12] are closer to the results of this work compared to the other literature. The residual thermodynamic method does not have the limitations of Clausius-Clapeyron and Clapeyron approaches. The scheme has more capabilities like the ability for easy calculation of enthalpies of hydrate phase transitions for other phase transitions like for instance, in case of hydrate forming from aqueous solution, and it can straightforwardly be extended to conditions outside of equilibrium as well as to other hydrate phase transitions

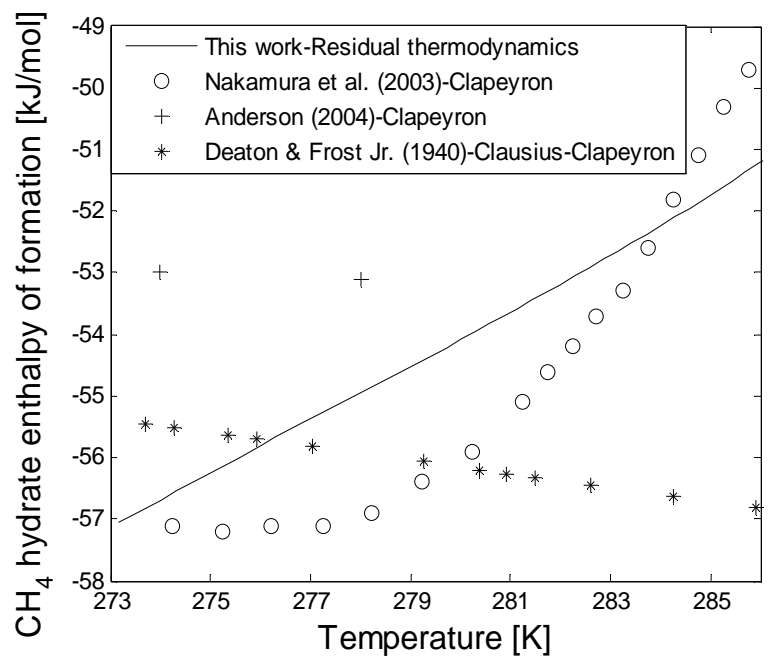

Figure 2: Estimated enthalpies of methane hydrate formation using residual thermodynamics (this work), Clapeyron equation [9, 15], and Clausius-Clapeyron equation [8].

\begin{tabular}{cccccc}
\hline \multicolumn{2}{c}{ Clapeyron equation (Nakamura et al. (2003)) } & \multicolumn{3}{c}{ Residual thermodynamics (This work) } \\
\hline $\begin{array}{c}\text { Temper- } \\
\text { ature [K] }\end{array}$ & $\begin{array}{c}\text { Pressure } \\
\text { [bar] }\end{array}$ & $\begin{array}{c}\text { Enthalpies of dissoci- } \\
\text { ation [kJ/mol] }\end{array}$ & $\begin{array}{c}\text { Temper- } \\
\text { ature [K] }\end{array}$ & $\begin{array}{c}\text { Pressure } \\
\text { [bar] }\end{array}$ & $\begin{array}{c}\text { Enthalpies of for- } \\
\text { mation [kJ/mol] }\end{array}$ \\
\hline 274.25 & 29.2 & 57.1 & 274.24 & 28.2 & 56.6 \\
275.25 & 32.2 & 57.2 & 275.24 & 31.4 & 56.1 \\
276.22 & 35.5 & 57.1 & 276.19 & 34.7 & 55.7 \\
277.24 & 39.2 & 57.1 & 277.26 & 38.9 & 55.3 \\
278.24 & 43.3 & 56.9 & 278.24 & 43.1 & 54.9 \\
279.23 & 47.9 & 56.4 & 279.21 & 47.8 & 54.4 \\
\hline
\end{tabular}

Table 1: Enthalpies of methane hydrate formation or dissociation [7]. 


\begin{tabular}{|c|c|c|c|c|c|}
\hline & Method & $\begin{array}{c}\text { Temperature } \\
{[\mathrm{K}]}\end{array}$ & $\begin{array}{c}\text { Pressure } \\
\text { [bar] }\end{array}$ & $\begin{array}{l}\text { Enthalpy of formation/ } \\
\text { dissociation }[\mathrm{kJ} / \mathrm{mol}]\end{array}$ & $\begin{array}{c}\text { Hydration } \\
\text { number (n) }\end{array}$ \\
\hline & & 273.15 & 25.19 & -57.07 & 6.46 \\
\hline & \multirow[b]{2}{*}{$\begin{array}{l}\text { Residual Thermo- } \\
\text { dynamics }\end{array}$} & 274.10 & 27.82 & -56.64 & 6.43 \\
\hline This work & & 278.02 & 42.15 & -54.94 & 6.35 \\
\hline \multirow[b]{2}{*}{ Anderson, G. K. (2004) } & \multirow{2}{*}{$\begin{array}{l}\text { Clapeyron equa- } \\
\text { tion }\end{array}$} & 274.00 & 28.50 & 53.00 & 5.89 \\
\hline & & 278.00 & 42.80 & 53.10 & 5.79 \\
\hline Deaton \& Frost (1946) & $\begin{array}{l}\text { Clausius- } \\
\text { Clapeyron equa- } \\
\text { tion }\end{array}$ & 273.15 & $\mathrm{n} / \mathrm{a}$ & 55.12 & $\mathrm{n} / \mathrm{a}$ \\
\hline Kang et al. (2001) & $\begin{array}{l}\text { Experiment-Iso- } \\
\text { thermal microcalo- } \\
\text { rimeter }\end{array}$ & 274.15 & $\mathrm{n} / \mathrm{a}$ & 56.84 & $\mathrm{n} / \mathrm{a}$ \\
\hline
\end{tabular}

Table 2: Enthalpies of hydrate formation and dissociation and hydration number [8, 13, 14].

\section{References}

[1] Aromada S A, Kvamme B. New approach for evaluating the risk of hydrate formation during transport of hydrocarbon hydrate formers of sI and sII. AIChE Journal 65(3), 1097-1110, (2019). DOI: 10.1002/aic.16493

[2] Kvamme B, Aromada S A. Risk of hydrate formation during the processing and transport of Troll gas from the North Sea. Journal of Chemical \& Engineering Data 62(7), 2163-2177 (2017). DOI: $10.1021 /$ acs.jced.7b00256

[3] Aromad S A, Kvamme B. Impacts of $\mathrm{CO}_{2}$ and $\mathrm{H}_{2} \mathrm{~S}$ on the risk of hydrate formation during pipeline transport of natural gas. Frontiers of Chemical Science and Engineering, 1-12 (2019). DOI: $10.1007 / \mathrm{s} 11705-019-1795-2$

[4] Kvamme B, Aromada S A, Saeidi N. Heterogeneous and homogeneous hydrate nucleation in $\mathrm{CO}_{2}$ /water systems. Journal of Crystal Growth, 522, 160-174 (2019).

[5] Anderson R, Llamedo M, Tohidi B, Burgass R W. Experimental measurement of methane and carbon dioxide clathrate hydrate equilibria in mesoporous silica. The Journal of Physical Chemistry B, 107(15), 3507-3514 (2003). DOI: $10.1021 / \mathrm{jp} 0263370$

[6] Kvamme B. Environmentally Friendly Production of Methane from Natural Gas Hydrate Using Carbon Dioxide. Sustainability 11(7), 1-23 (2019). DOI: $10.3390 /$ su11071964

[7] Gupta A, Lachance J, Sloan E D, Koh C A. Measurements of methane hydrate heat of dissociation using high pressure differential scanning calorimetry. Chemical Engineering Science, 63, 5848-5853 (2008). DOI: $10.1016 /$ j.ces.2008.09.002
[8] Deaton W M, Frost Jr E M. In: Proceeding of American Gas Association, 1940, 122, Houston, Texas, May 6 (1940). Cross referenced from [6]

[9] Anderson G K. Enthalpy of dissociation and hydration number of methane hydrate from the Clapeyron equation. The Journal of Chemical Thermodynamics, 36(12), 1119-1127, (2004).

DOI: $10.1016 /$ j.jct.2004.07.005

[10] Kvamme B, Tanaka H. Thermodynamic stability of hydrates for ethane, ethylene, and carbon dioxide. The Journal of Physical Chemistry, 99(18), 7114-7119, (1995).

[11] Kvamme B, Kuznetsova T, Stensholt S, Sjöblom S. Investigations of the Chemical Potentials of Dissolved Water and $\mathrm{H}_{2} \mathrm{~S}$ in $\mathrm{CO}_{2}$ Streams Using Molecular Dynamics Simulations and the Gibbs-Duhem Relation. Journal of Chemical \& Engineering Data, 60(10), 2906-2914 (2015) DOI: $10.1021 /$ acs.jced.5b00267

[12] Kvamme B, Førrisdahl O K. Polar guest-molecules in natural gas hydrates. Fluid Phase Equilibria, 83, 427-435, (1993). DOI: 10.1016/0378-3812(93)87047-5.

[13] Nakamura T, Makino T, Sugahara T, Ohgaki K. Stability boundaries of gas hydrates helped by methane-structure$\mathrm{H}$ hydrates or methylcyclohexane and cis-1,2-dimethylcyclohexane. Chemical Engineering Science, 58(2), 269-273. (2003).

DOI: 10.1016/S0009-2509(02)00518-3

[14] Deaton W M, Frost Jr E M. Gas hydrate composition and equilibrium data. Oil Gas Journal, 45, 170-178 (1946). Cross referenced from [6] 
[15] Kang S P, Lee H, Ryu B J. Enthalpies of dissociation of clathrate hydrates of carbon dioxide, nitrogen, (carbon dioxide + nitrogen), and (carbon dioxide + nitrogen + tetrahydrofuran). The Journal of Chemical Thermodynamics, 33(5), 513-521 (2001). DOI: 10.1006/jcht.2000.0765.

[16] Kvamme B, Aromada S A, Gjerstad P B. Consistent enthalpies of the hydrate formation and dissociation using residual thermodynamics. Journal of Chemical \& Engineering Data, 64(8), 3493-3504 (2019).

[17] Aromada S A, Kvamme B, Wei N, Saeidi N. Enthalpies of hydrate formation and dissociation from residual thermodynamics. Energies, 12(24), 4726 (2019).

[18] Aromada S A, Kvamme B. Production of Methane from Hydrate and $\mathrm{CO}_{2}$ Zero-Emission Concept. Presented at the 10th EUROSIM2019 Congress, Logroño (La Rioja), Spain, Preprint: 1-5 (2019, July).
[19] Kvamme B. Kinetics of hydrate formation, dissociation and reformation. Chemical Thermodynamics and Thermal Analysis, 1-2, 100004, March (2021).

[20] Kvamme B, Clarke M. Hydrate Phase Transition Kinetic Modeling for Nature and Industry-Where Are We and Where Do We Go? Energies, 14(14), 4149 (2021).

[21] Kvamme B. Enthalpies of hydrate formation from hydrate formers dissolved in water. Energies, 12(6), 1039 (2019).

[22] Kvamme B, Zhao J, Wei N, Sun W, Saeid N, Pei J, Kuznetsova T. Hydrate production philosophy and thermodynamic calculations. Energies, 13(3), 672 (2020).

[23] Svandal A. Modeling Hydrate Phase Transitions Using Mean-Field Approaches [PhD dissertation], Bergen, Norway: University of Bergen, (2006). 